Trauma Berufskrankh 2003 · [Suppl 1] : S7 DOI 10.1007/s10039-003-0813-1 Online publiziert: 27. November 2003 (c) Springer-Verlag 2003
B. Fellmer

Landesverband Südwestdeutschland der gewerblichen Berufsgenossenschaften, Heidelberg

\section{Eröffnung der Unfallmedizinischen Tagung 2003}

themen sind zudem geeignet, Informationen für die Zusammenarbeit zwischen Ärzten und Unfallversicherungsträgern zu geben und Wege zu weisen, diese $\mathrm{Zu}$ sammenarbeit noch effizienter zu gestalten.

Auch die diesjährige Tagung scheint mir geeignet, die gemeinsame Arbeit erneut unter Beweis zu stellen. Neue unfallmedizinische Erkenntnisse und die sich für Krankenhäuser, Kostenträger und die Versicherten aus dem neuen Fallpauschalengesetz ergebenden vielfältigen Probleme machen deutlich, dass noch mehr als bisher die Notwendigkeit besteht, im Dialog nach Möglichkeiten zu suchen, die auch künftig Humanität und Ökonomie sichern. Die Realisierung dieser Zielvorstellung ist sicherlich nicht leicht. Unsere Bemühungen und Erfolge in diesem Bereich der Vergangenheit sollten Antrieb bleiben, diesen Weg, ich betone, diesen gemeinsamen Weg, beharrlich weiter zu gehen. Mit diesem durchaus hoffnungsfrohen Ausblick schließt sich zugleich der Kreis meiner Gedanken zu Beginn der diesjährigen Unfallmedizinischen Tagung unseres Landesverbands.

Zum Abschluss sei noch einigen Personen gedankt: Mein besonderer Dank geht zunächst an die wissenschaftlichen Leiter der Tagung, die Herren Professoren Weise und Wentzensen, an die Damen und Herren, die den Vorsitz zu den Hauptthemen oder die Moderation übernommen haben und nicht zuletzt an die Da- men und Herren, die sich bereit erklärt haben, Referate zu halten.

Ihnen allen wünsche ich, dass Sie trotz des umfangreichen Tagungsprogramms auch Zeit finden mögen, hier in BadenBaden einige anregende und schöne Stunden zu verbringen. Mit unserem Rahmenprogramm möchten wir dazu beitragen. So darf ich die Nichtkongressteilnehmer noch einmal darauf hinweisen, dass am heutigen Nachmittag um 14.00 Uhr die Möglichkeit besteht, das Schloss „Favorite“ bei Rastatt zu besichtigen. Die Abfahrt mit Omnibus findet ab Kongresshaus Augustaplatz statt. Rückkehr wird um 17.00 Uhr sein.

Heute Abend findet um 20.30 Uhr hier, an gleicher Stelle, unser traditioneller Gesellschaftsabend mit Tanz und einem attraktiven Rahmenprogramm statt. Auch hierzu sind Sie alle herzlich eingeladen.

Hiermit eröffne ich die 30. Unfallmedizinische Tagung des Landesverbandes Südwestdeutschland der gewerblichen Berufsgenossenschaften und lege deren weitere Leitung in die Hände der wissenschaftlichen Leiter.

\section{Korrespondierender Autor} B. Fellmer

Landesverband Südwestdeutschland der gewerblichen Berufsgenossenschaften, Kurfürstenanlage 62,69115 Heidelberg E-Mail: rsaenger@|vbg.de len und Probleme aufzuwerfen, sondern auch Lösungsmöglichkeiten für die Praxis aufzuzeigen. So genannte Verwaltungs- 